\title{
COMMUNICATIONS
}

\section{HEREDITARY DEGENERATION OF THE MOUSE RETINA*}

\author{
BY \\ KATHARINE TANSLEY \\ Institute of Ophthalmology, London
}

TRUE hereditary degeneration of the retina has been described in the rat (Bourne, Campbell, and Tansley, 1938) and the dog (Hodgman and others, 1949), but certainly not in any other species. . In a series of papers, Keeler $(1924,1926,1927,1928)$ described an inherited retinal anomaly in the house mouse which he called " rodless retina ". This anomaly is inherited as a Mendelian recessive character and the mice are blind by conditioned reflex tests. In addition, no electroretinogram could be obtained from them (Keeler and others, 1928). Keeler claims that the condition is one of arrested development, the retina developing normally until 6 or 7 days after birth but not thereafter progressing beyond the separation of the inner and outer fibre layers. No rods ever develop, and there is said to be a reduction of normal mitotic activity from birth onwards (foetal eyes were not examined) and no degeneration. Such a condition could obviously not be considered as a retinal degeneration.

Cohrs $(1933 a, b)$ described several mice with a retinal lesion very like Keeler's rodless retina. He was unable to obtain any data as to its inheritance but, on the basis of the histological picture, identified the condition as rodless retina. Grüneberg (1943), however, considers Cohrs's lesion more likely to be homologous with the hereditary degeneration of the rat.

The stock of mice described here, which was obtained from Dr. Brückner through the kindness of Professor Sorsby, is an albino stock carrying the retinal lesion as a recessive Mendelian character. No other abnormality has been found in the stock. No cataracts have been observed although seven generations have been bred at the Institute of Ophthalmology and some animals have survived for well over a year. There is no known genetic relationship between this stock and those investigated by Keeler, Cohrs, or Hopkins (1927), although the possibility (particularly where Cohrs's and Hopkins' mice are concerned) cannot be ruled out (Brückner, 1951).

\footnotetext{
* Received for publication June 14, 1951.
} 


\section{MATERIAL AND TECHNIQUe}

The eyes of 60 animals were examined microscopically at ages varying from birth to 15 months. The younger animals were killed by knocking on the head, the older by breaking the neck. The eyes were removed immediately after death and fixed in Zenker's solution.

Paraffin sections of the back of the eyeball were cut at $6 \mu$ and stained with haematoxylin and eosin, or with the azan modification of Mallory's triple connective tissue stain (in a few cases Mallory's original method was used). Sections from older animals were also stained with Mallory's phosphotungstic acid haematoxylin for glial fibres. In addition, a few eyes were stained by Feulgen's method, and a few with Heidenhain's iron haematoxylin counterstained with an aniline blue and orange $G$ mixture.

All eyes from the affected stock up to weaning age ( 21 days) were compared with eyes from two normal stocks of the same ages.

\section{RESULTS}

As in rats, the eyes of mice are immature at birth, the retina being then composed of a ganglion cell layer, an inner fibre layer, and a thick layer of undifferentiated cells showing mitosis along the outer edge (Fig. 1).

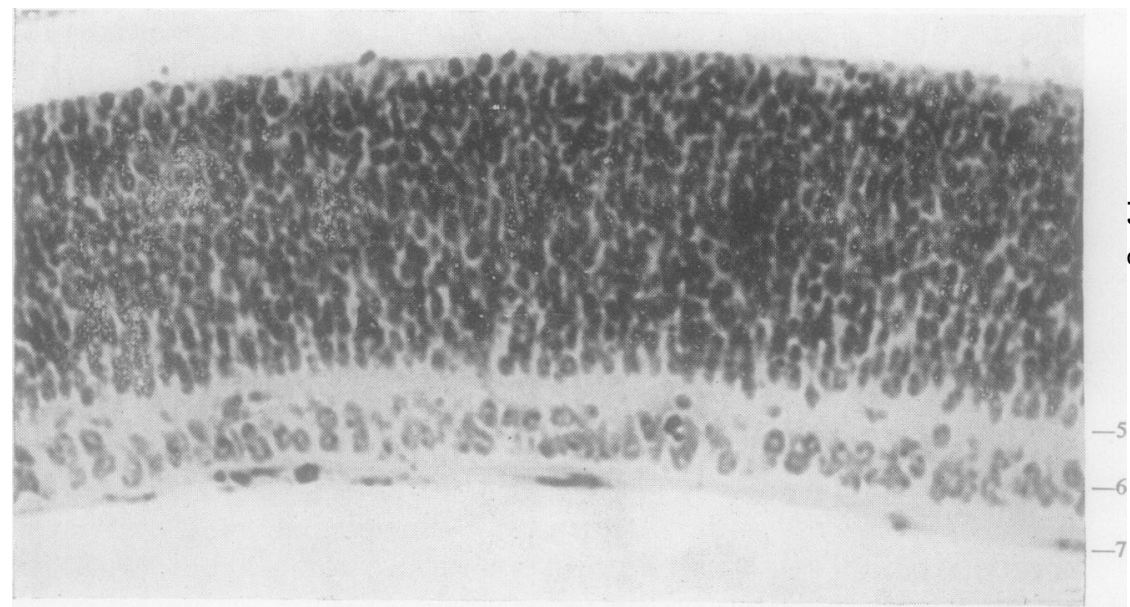

FIG. 1.-Transverse section through the mouse retina at birth. Zenker. Haematoxylin and eosin. $(\times 240)$.

The following numbering applies to all Figures: 1. Rods. 2. Outer nuclear layer. 3. Outer fibre layer. 4. Inner nuclear layer. 5. Inner fibre layer. 6. Ganglion cells.
7. Optic nerve fibres.

In the affected stock, the retina appeared to develop normally until the animals were about 14 days old. Differentiation of the inner nuclear layer (ordinarily visible with this fixation method at about 4 days) was a little late compared with the two normal stocks, and so was the first appearance of both inner and outer limbs of the rods ( 7 and 10 days respectively in the normal stocks). However, at 13 days the retina was well developed and looked healthy (Fig. 2). 


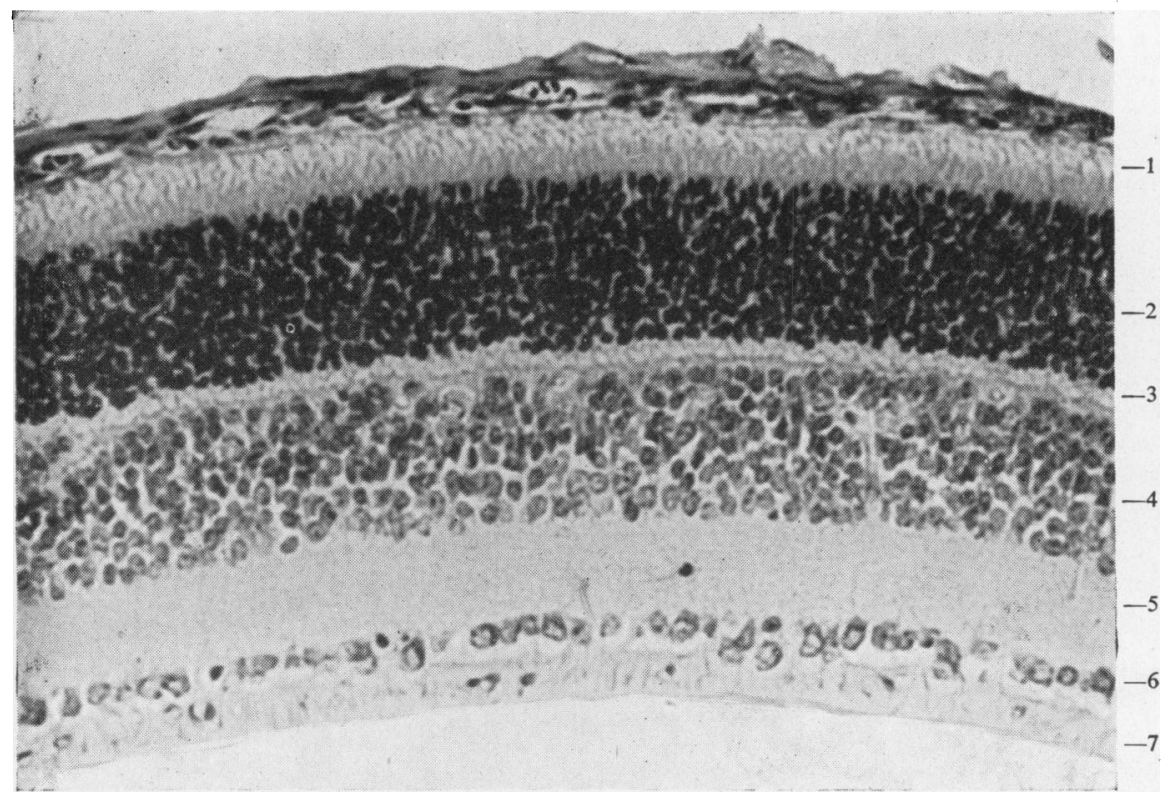

FIG. 2.- Section through the retina of a mouse aged 13 days, from the affected stock. The retina is normal for an animal of this age. Zenker. Haematoxylin and eosin. $(\times 240)$.

The first retinal changes were seen in two out of three animals examined at 14 days. From 14 days . until about 4 weeks the condition was always bilateral and progressed at about the same rate in both eyes and, so far as could be told, in different animals.

The general course of the disease was very much like that already described in rats, although there was some difference in details. The lesion appeared earlier and progressed much more rapidly in the mouse, but this may well be nothing more than an expression of the shorter life and quicker metabolism of the mouse. As in the rats, the first sign of abnormality was the appearance of pyknotic cells in the outer nuclear layer of the central retina. These degenerations could be seen at 14 days; by 15 days they had spread to the periphery and the outer nuclear layer was obviously thinner than normal in the centre. Fig. 4 shows the appearance of the central retina at 16 days, Fig. 3 being from a normal mouse of 17 days for comparison. In the affected animals the rods remained fairly normal in appearance over the 15 th day, but between the 16th and 19th days they gradually lost their characteristic structure. On the 21st day nothing but debris could be seen between the external limiting membrane and the pigment epithelium, and at 4 weeks there was no sign of the rods or, except here and there, of their nuclei (Fig. 5).

Also at 4 weeks the external limiting membrane could be seen only in places, and part of the pigment epithelium began to look swollen and abnormal. From this stage onwards the rate of progress of the lesion 


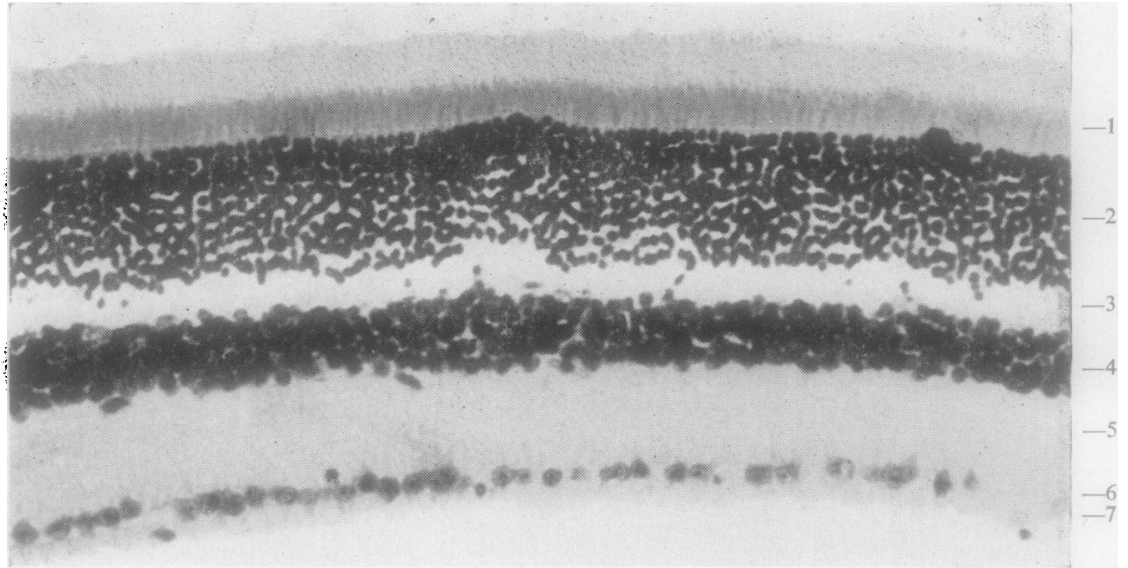

Fig. 3.-Section through the normal fully-developed mouse retina

(17 days). Zenker. Haematoxylin and eosin. ( $\times 240)$.

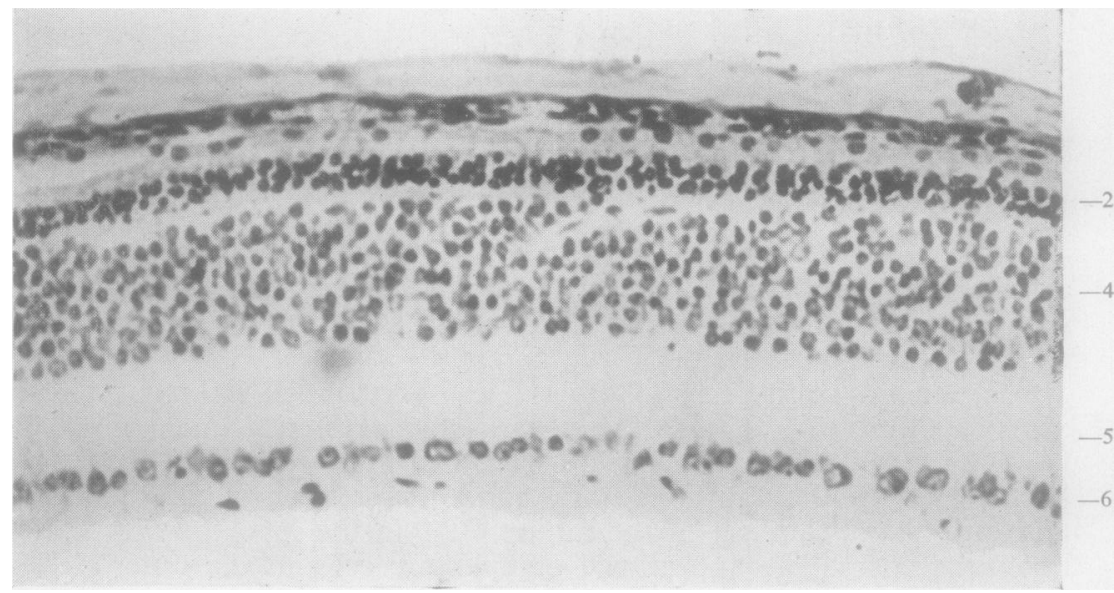

Fig. 4.-Section through the retina of a mouse aged 16 days from the affected stock. The outer nuclear and rod layers are much reduced.

Zenker. Haematexylin and eosin. $(\times 240)$.

became more irregular, some retinae becoming seriously disorganized fairly quickly, others much more slowly. In addition, different parts of a given retina were often at slightly different stages. Not much change was seen between 5 and 8 weeks except for slight gliosis and uneven swelling or disappearance of the pigment epithelium, which might, however, still look fairly normal in places. At 9 weeks the choroid also began to look unhealthy, varying between an enormous sclerosed thickening and almost complete disappearance. In every retina examined at this stage, however, there were always places where both the pigment epithelium and 


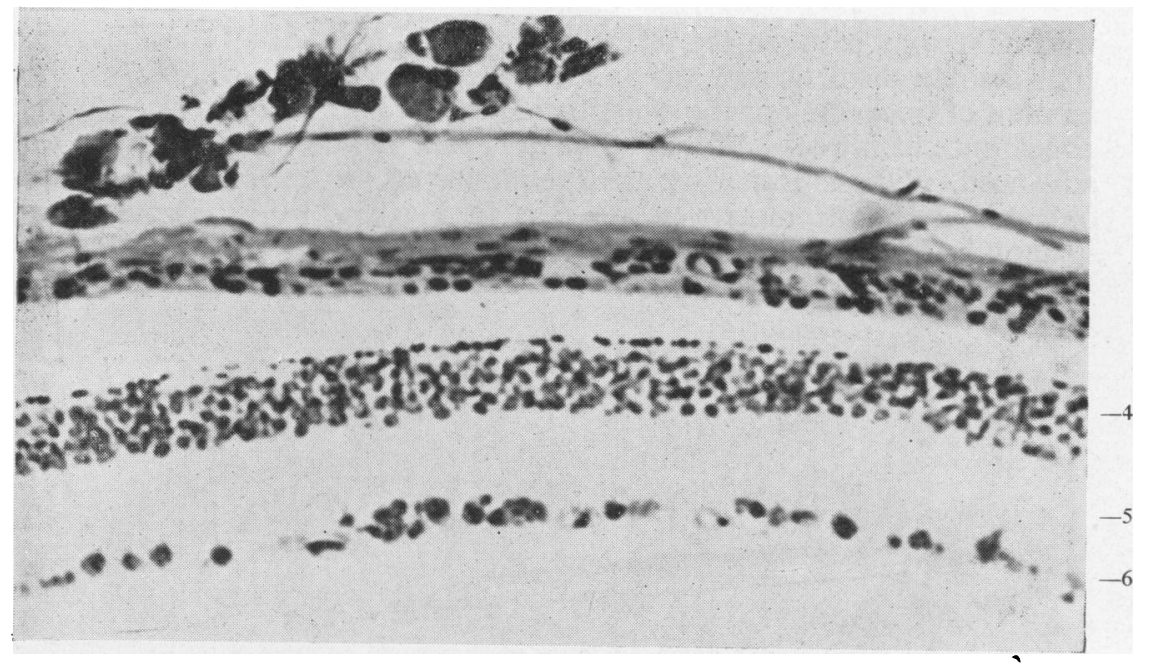

Fig. 5.-Section through the retina of a mouse aged 5 weeks, from the affected stock. Where the outer nuclear layer is present it is reduced to a single layer of abnormal cells. Zenker. Haemat $x$ xylin $\cong$ nd eosin. $(\times 240)$.

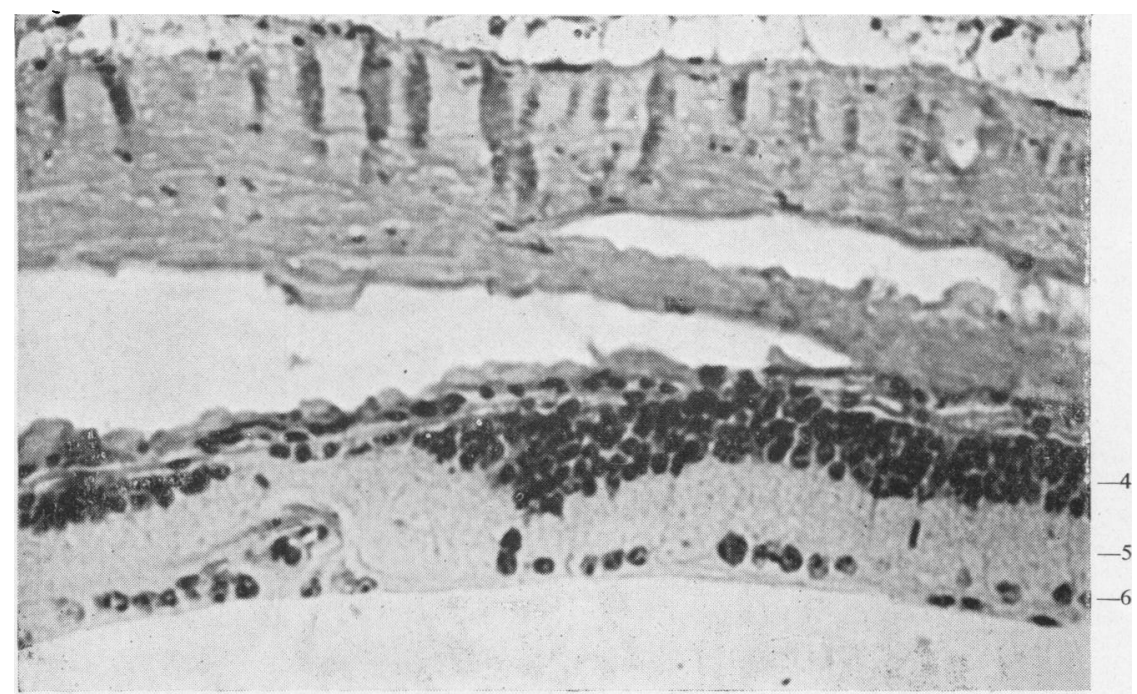

Fig. 6.-Section through the retina of a mouse aged 11 months, from the affected stock. The inner nuclear layer is disorganized and there is adhesion between the retina and the back of the eyeball. Zenker. Haematoxylin and eosin. $(\times 240)$.

the choroid looked fairly normal. From about 4 months, connective tissue fibres and vessels could be seen running right through between the inner retinal surface and the choroid. These were, of course, associated 
with adhesions between the retina and choroid (Fig. 6) and usually with considerable distortion of the inner nuclear layer (Figs 6 and 7). The amount of thickening of the glial fibres was very variable, and after its first appearance at 6 weeks did not seem to depend on how far the lesion had advanced. Gliosis is marked (for the mouse) in Fig. 8, from a 12-monthold animal, but is much less obvious in Figs 6 and 7, from 11- and 13-month-old mice respectively.

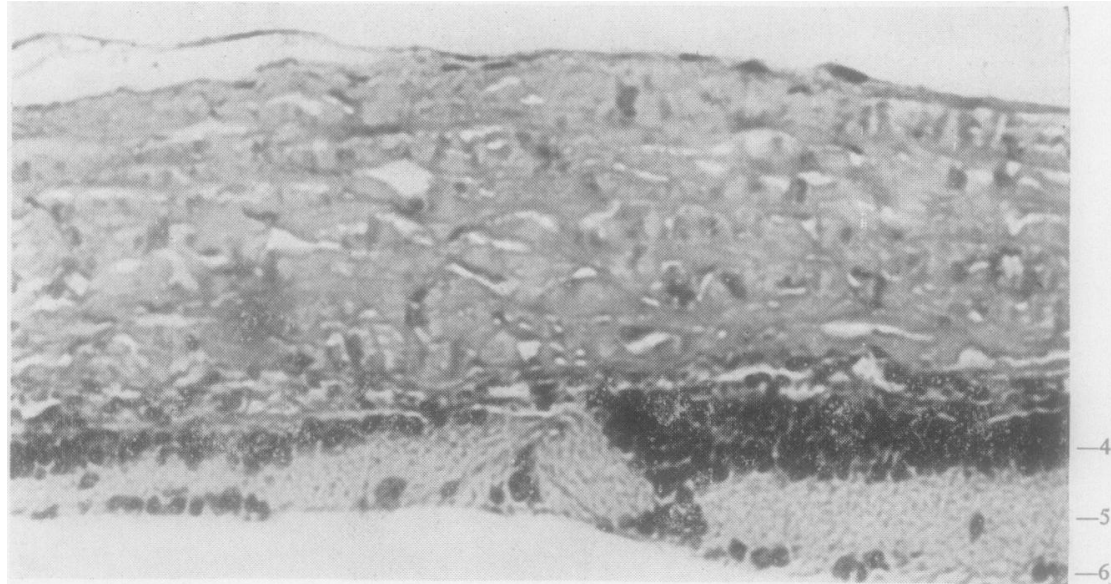

Fig. 7.-Section through the retina of a mouse aged 13 months, from the affected stock. Note the disorganization of the inner retinal layers associated with a bundle of connective-tissue fibres joining the retina to the choroid. Zenker. Haematoxylin and eosin. ( $\times 1240)$.

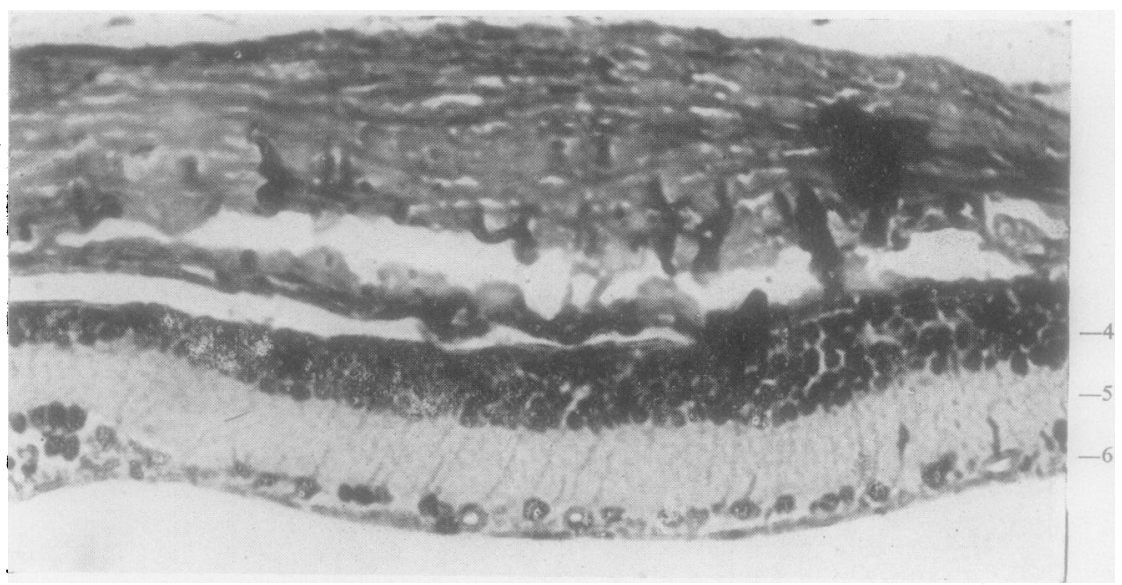

FIG. 8.-Section through the retina of a mouse aged 12 months, from the affected stock, showing gliosis but very little distortion of the inner retinal layers as well as separation of the retina from the choroid. Zenker. Mallory's phosphotungstic acid haemotoxylin. $(\times 240)$. 


\section{Discussion}

The changes which result in the disappearance of the visual cells are constant and regular, and appear to be a fairly direct manifestation of the effect of the mutant gene. The later changes, affecting the pigment epithelium, the choroid, and the inner part of the retina, are much more variable in appearance; they are probably secondary to the destruction of the rods and so only indirectly related to the fundamental genetic abnormality. We shall see later that removal of the visual cells and their nuclear layer, from whatever cause, tends to produce retinal and choroidal changes of this sort. This type of disturbance is often associated with lesions which result in the breaking of the normal barrier between the retina and choroid. Since these are albino animals there is, of course, no question of pigment migration into the retina such as we observed in the rats.

The whole picture and course of the disease suggests that we are here dealing with the mutation that causes hereditary retinal degeneration in rats and perhaps dogs, and retinitis pigmentosa in man.

Cogan (1950), distinguishes two retinal conditions in man which he calls retinitis pigmentosa and pseudoretinitis pigmentosa. According to his description, although in some parts of the retina the histological picture in one condition may be indistinguishable from that in the other, the two show a distinct over-all difference. In pseudoretinitis pigmentosa the inner layers are more disorganized and the gliosis is more marked. But the most important difference between the two lesions is that in pseudoretinitis pigmentosa there is a more extensive, although usually focal, disappearance of the pigment epithelium and a firm union between retina and choroid. On the other hand, according to Cogan (1950) the fusion of retina and choroid in retinitis pigmentosa is usually of a tenuous kind, so that the retina easily becomes detached in histological preparations, whereas in pseudoretinitis pigmentosa it remains adherent to the choroid. There may also be considerable fibrosis of the choroid in the latter condition. Cogan accepts the original suggestion of Collins (1919) that retinitis pigmentosa is due to an abiotrophy, but he suggests that pseudoretinitis pigmentosa has some other aetiology, which may possibly be inflammatory.

Now, although some of Cogan's illustrations of pseudoretinitis pigmentosa (notably Figs 34 and 35) do not resemble any stage of the mouse lesion described here, his description of the histological differences between the two conditions would, in general, do very well as a description of the individual differences found during the later stages of the mouse disease. For instance, in the retina illustrated in Fig. 8, the inner layers are not seriously disorganized and there is no real adhesion between retina and choroid. In the retinae shown in Figs 6 and 7 there is considerable disturbance of the inner layers and, in Fig. 6 at least, there has been such a firm union between 
the choroid and retina that the separation during histological preparation has brought away part of the back of the eyeball with the retina. These two pathological pictures are both found as part of the same retinal condition, one which Treacher Collins would certainly have called an abiotrophy. It is very doubtful, therefore, whether adhesion or non-adhesion of the retina and choroid can be regarded as a distinguishing feature between diseases of an inflammatory and of a genetic origin.

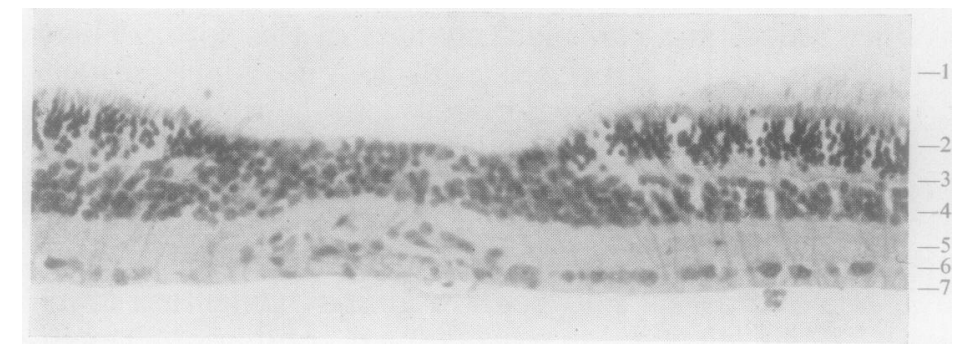

FIG. 9.-Section through an adult rabbit retina, showing a small area where rods, cones, and outer nuclear layer have disappeared. Zenker. Haematoxylin and eosin.

Cogan (1950) describes one case of what he called " focal pseudoretinitis pigmentosa", in which the rods, cones, and outer nuclear layer are missing over relatively small areas only. I have seen a similar lesion in several rodent species, mouse, rat, rabbit (Mann and others, 1946), and ferret (Thomson, 1951), and Parry (Hodgman and others, 1949) has observed it in the dog. I have not-had the opportunity of doing satisfactory inheritance studies on this lesion, but I am inclined to think that it is familial and progressive, at least in the rat, and Parry believes the same may be true in the dog. Fig. 9 shows a section through an affected rabbit retina, and the similarity to Cogan's Fig. 36 is most striking. I believe that Fig. 9 illustrates an early stage of the disease and that it may progress to give the picture seen in Fig. 10. This and Fig. 11 are from different parts of the same section through the retina of an affected rat, and Fig. 11 might well be from an animal with the hereditary retinal lesion described here. This latter disease, whether in rats, mice, or dogs, never produces a loss of retinal tissue as severe as that illustrated in Fig. 10, nor does one ever see such large differences within one eye. In making a differential diagnosis between these two conditions, hereditary degeneration on the one hand and focal degeneration on the other, therefore, it is absolutely necessary to examine the whole retina, not just a few sections, and to catch the disease in its early stages if this is at all possible. The evidence from animal material strongly suggests that these two retinal degenerations are unrelated. 


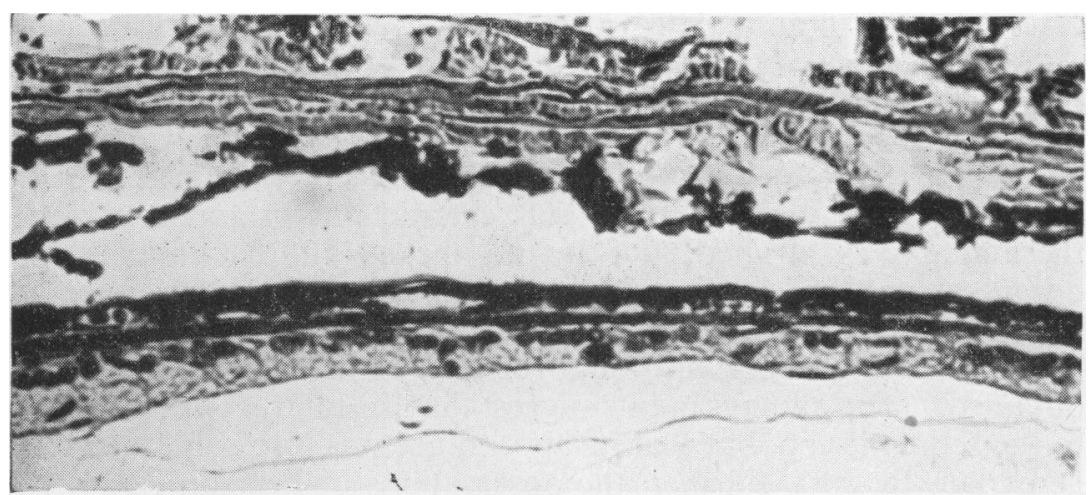

FIG. 10.-Section through a rat retina showing such extensive destruction that the retina is reduced to a single layer of nuclei and some fibres. There is adhesion between the retina and choroid. Zenker. Iron haematoxylin, aniline blue, and orange G. $(\times 240)$.

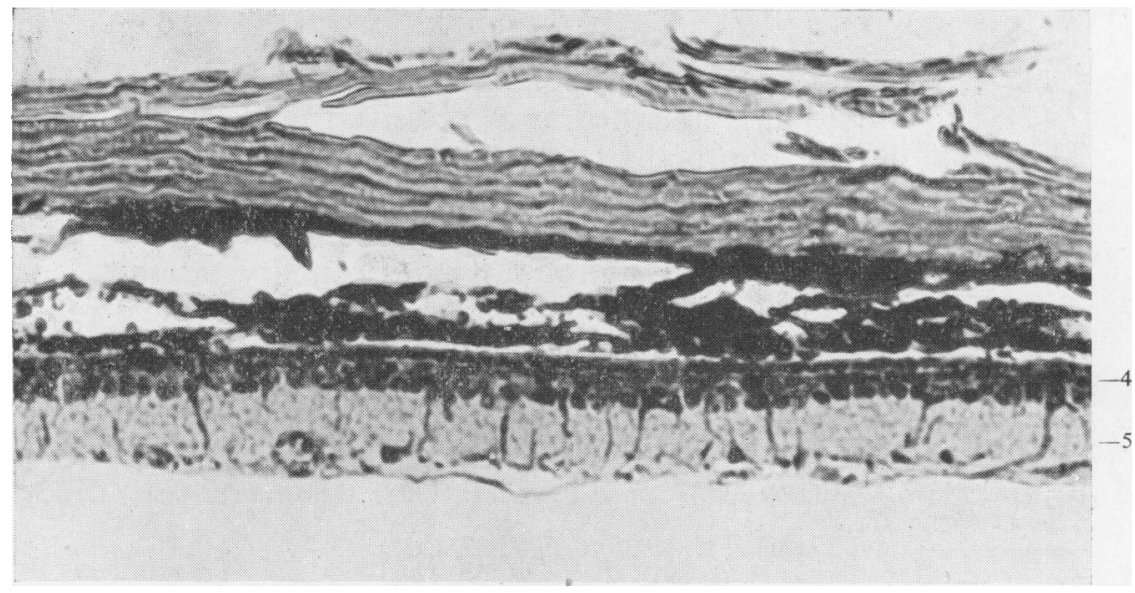

FIG. 11.-Another part of the section illustrated in Fig. 10. Part of the inner nuclear layer is still present with the inner fibre and ganglion cell layers. Gliosis is also present (cf. Fig. 8). Zenker. Iron haematoxylin, aniline blue, and orange G. $(\times 240)$.

Hereditary retinal degeneration is often associated with cataract in the rat (Bourne and others, 1938a; Bourne and Grüneberg, 1939), and the dog (Hodgman and others, 1949), and retinitis pigmentosa is often associated with cataract in man. This association of lens and retinal changes appears not to have been seen so far in mice. As already mentioned, no member of the mouse stock described here has ever developed any recognizable lens opacity, and it seems that neither Keeler, Hopkins, nor Cohrs obs ved cataracts in their animals.

The absence of cataracts in mice with hereditary retinal degeneration 
cannot be due to an inability of mice to develop lens opacities, since, although reports of mouse cataracts are rare, the condition has been observed by Pearson (1924). The incidence of his cataract was increased from 1.5 to nearly 50 per cent. by selection (Grüneberg, 1943), but the retinae of these animals were not examined. In the rat stock investigated by us in 1938, cataracts never appeared unless the retina was affected, but by no means all the animals with degenerate retinae produced cataracts. As in Pearson's mice the incidence of cataract in these rats was greatly increased by selective breeding, but a stock showing 100 per cent. cataract was never obtained. The findings in rats suggest that the cataract was secondary to the retinal lesion, but that its appearance was also subject to some hereditary factor. Further, the observations of Pearson (1924) and Grüneberg (1943) on mice suggest a cataract subject to a hereditary factor but not a direct expression of the mutant gene (one with a relatively low penetrance). It may well be that this second mutant gene is not present in the mouse stock described in this paper.

\section{SUMMARY}

(1) A hereditary degeneration of the retina in mice is described.

(2) The condition is inherited as a recessive Mendelian character. The retina develops normally to the adult stage when the rod nuclei degenerate. Loss of outer layers of the retina follows, and at a later stage there is disorganization of the inner layers and adhesion between retina and choroid. There is no secondary cataract.

(3) The relationship of this lesion in the mouse to some retinal degenerations in other animals and in man is discussed.

The author is indebted to the Medical Research Council for a personal grant.

\section{REFERENCES}

Bourne, M. C., Campbell, D. A., and Pyke, M. (1938). British Journal of Ophthalmology, 22, 608.

and TANSLEY, K. (1938). Ibid., 22, 613. and GRüNEBERG, H. (1939). J. Hered., 30, 131.

BRÜCKNER (1951). Personal communication.

CogAN, D. G. (1950). Trans. Amer. Acad. Ophthal. Otolaryng., 54, 629.

CoHRS, P. (1933a). Arch. Augenheilk., 107, 489. (1933b). Dtsch. tierärztl. Wschr., 41, 549.

Collins, E. TREACHER (1919). Trans. ophthal. Soc. U.K., 39, 165.

GRÜNEBERG, H. (1943). "The Genetics of the Mouse ", University Press, Cambridge.

Hodgman, S. F. J., Parry, H. B., Rasbridge, W. J., and Śteel, J. D. (1949). Vet. Rec., $61,185$.

Hopkins, A. E. (1927). Proc. nat. Acad. Sci. Wash., 13, 488.

KEELER, C. E. (1924). Ibid., 10, 329.

- (1926). Ibid., 12, 255 .

(1927). J. exp. Zool., 46, 355.

(1928). Ibid., 51, 495.

Sutcliffe, E., and Chaffee, E. L. (1928). Proc. nat. Acad. Sci. Wash., 14, 477.

MANN, I., PIRIE, A., TANSLEY, K., and WoOd, C. (1946). Amer. J. Ophthal., 29, 801.

PARRY, H. B., see Hodgman, S. F. J., and others (1949).

Pearson, E. S. (1924). Nature, Lond., 114, 433.

Thomson, A. P. D. (1951). J. Physiol., 113, 425. 